\title{
Preference for a segmented schedule using a brief S+ stimulus correlated with a great delay reduction in humans
}

\author{
Jérôme Alessandri ${ }^{\mathrm{a}, *}$, Mikaël Molet ${ }^{\mathrm{a}}$, Edmund Fantino ${ }^{\mathrm{b}}$ \\ a University of Lille, North of France, France \\ ${ }^{\mathrm{b}}$ University of California, San Diego, CA, United States
}

\section{A R T I C L E I N F O}

\section{Article history:}

Received 17 November 2009

Received in revised form 25 May 2010

Accepted 7 June 2010

\section{Keywords:}

Concurrent-chain

Delay-reduction

Humans

Preference

Segmentation

\begin{abstract}
A B S T R A C T
Past studies using the concurrent-chain procedure showed that pigeons and humans generally prefer an unsegmented schedule to a segmented schedule. This finding is ostensibly inconsistent with theories of conditioned reinforcement such as delay-reduction theory. In the present study with humans, two changes in the basic segmented schedule were implemented to resolve this inconsistency. The first change was that in the segmented schedule the terminal-link stimulus ( $\mathrm{S}+$ stimulus) changed late in the terminallink, close to reinforcement presentation. The second change was that the presentation of the segmenting stimulus, $\mathrm{S}+$, was brief allowing a reinstatement of the early terminal-link stimulus, which is contiguous with reinforcement. Our data constitute the first demonstration of preference for the segmented schedule when a brief $\mathrm{S}+$ is correlated with a greater reduction in delay to reinforcement.
\end{abstract}

(C) 2010 Elsevier B.V. All rights reserved.

\section{Introduction}

Imagine that you must wait a prolonged period to obtain a reward. Would you prefer a situation in which no temporal cue occurs before the arrival of reward or would you prefer a single cue sometime during the interval indicating how much time remains? For example, without cue, you might spend the entire duration of 30 min without any external cue that indicates how much time remains. With-cue, after $25 \mathrm{~min}$ a friend tells you that you will receive the reward in $5 \mathrm{~min}$. A rich literature on conditioned reinforcement suggests that a with-cue outcome would be preferred. This is because according to delay-reduction theory (DRT; Fantino, 1969,2008 ) the verbal cue is correlated with an $83 \%$ ( 25 of $30 \mathrm{~min}$ ) reduction in time to reward and might therefore function as an effective conditioned reinforcer, thereby enhancing preference.

However, from a different perspective, based on research on schedule segmentation, the opposite prediction might be made because the verbal cue segments the temporal interval, and typically an unsegmented outcome is preferred (Duncan and Fantino, 1972; Leung and Winton, 1986, 1988, all with pigeons; Leung, 1989, 1993 with humans). Research on segmentation and preference has been conducted with concurrent-chain schedules developed by Autor $(1960,1969)$ and Herrnstein (1964) in which preference for two outcomes is measured by responses in the choice phase (during the so-called initial links). Since the two schedules in the choice

\footnotetext{
* Corresponding author at: Department of Psychology, University of Lille Nord de France, rue du Barreau, BP 60149, 59653 Villeneuve d'Ascq Cedex, France.

E-mail address: jerome.alessandri@univ-lille3.fr (J. Alessandri).
}

phase are typically equal, any difference in responding to them may be attributed to preference for the respective following outcomes (the so-called terminal-links).

Is preference for the unsegmented outcome due to a lack of segmentation per se, namely due to a preference for one segment during the unsegmented outcome as opposed to two or more segments for the segmented outcome? Or is preference for the unsegmented outcome due to the fact that the stimulus obtained at the onset of the unsegmented outcome is the same as the stimulus present when the reinforcer is obtained whereas the stimulus obtained at the onset of the segmented outcome is different (at least in the typical segmented schedule where the terminal-link starts with one stimulus and ends with another)? In effect, in this case, it looks like a self-control situation involving conditioned reinforcement. The unsegmented schedule stands for the impulsive alternative because following a choice response, a lower valued conditioned reinforcer is presented (lower valued because its onset is relatively far from food delivery). And the segmented alternative stands for the self-control alternative because following a choice response, a higher valued conditioned reinforcer presentation is delayed (higher valued because its onset is relatively closer to reinforcement and the earlier stimulus has no or relatively little conditioned value because it is not contiguous with food). The present experiment explores these questions with human participants. In one condition, similar to the typical segmented schedule, the stimulus at the onset of the segmented outcome differs from the stimulus that precedes reinforcement. However in another condition, the stimulus in the beginning of the outcome is the same as the stimulus at the end of the outcome, just before reinforcement, with a different stimulus being presented at some point during the 
outcome (called here the S+ stimulus). We might expect a diminution or elimination of preference for the unsegmented outcome in this condition because in the segmentation alternative the early stimulus is now contiguous with reinforcement and would have the same value as the terminal-link stimulus of the unsegmented alternative.

There are three hypotheses implicit in this discussion. (1) Number of Segment Hypothesis (NSH): This hypothesis proposes that preference is inversely related to the number of segments in a given outcome. According to NSH, the unsegmented outcome should be preferred with the reinstatement outcome least preferred (since it has three segments). (2) Reinstatement Hypothesis (RH): According to the reinstatement hypothesis, the unsegmented outcome is preferred since the stimulus produced upon entry into the outcome is also contiguous with reinforcement. If so, preference should also occur in the segmented outcome in which the stimulus produced upon entry into the outcome is reinstated before (and therefore is contiguous with) reinforcement. Thus if the reinstatement hypothesis is sufficient, both the reinstatement outcome and the unsegmented outcome should be preferred to the conventional segmented outcome. (3) Delay-reduction Hypothesis (DRT): DRT predicts the value of a conditioned reinforcer based on the signaled delay reduction of overall time to the primary reinforcer. According to DRT, preference should be greatest for the segmented outcome with or without reinstatement since the S+ signals an $83 \%$ delay reduction in either case.

In the studies of segmentation cited above (e.g., Duncan and Fantino, 1972; Leung, 1989), stimulus change as a result of segmentation occurred midway through the terminal-link. Therefore, in terms of DRT, the stimulus change was correlated with a $50 \%$ reduction in time to reward. In contrast, Leung and Winton $(1986,1988)$ manipulated the temporal location of segmentation in several conditions with pigeons. When the S+ stimulus in the segmented outcome was relatively close to the reinforcement (and therefore correlated with greater delay reduction), preference for the unsegmented outcome tended to be less pronounced, thus was consistent with DRT. To further investigate the importance of temporal factors regarding the stimulus change, in the present experiment with humans, we compared conditions in which the intervening stimulus was correlated with $20 \%$ or $80 \%$ delay reduction (i.e., manipulating the segmentation ratio on preference relative to the unsegmented schedule). Here we might expect greater preference for the unsegmented outcome when the delay reduction correlated with the intervening stimulus of the segmented outcome was only $20 \%$, but preference for the segmented schedule when the S+ stimulus occurred after $80 \%$ of the outcome and the earlier stimulus was reinstated. With respect to the comparison of the $80 \%$ and $20 \%$ delay-reduction groups, the reinstatement and number of segments hypotheses predict an absence of difference in choice for the segmented schedule. For DRT, of course, the proportion of choice for the segmented schedule in the $80 \%$ group should be higher than in the $20 \%$ group.

Experiment 1 tested the effect of segmentation ratio (i.e., segmented at $80 \%$ or $20 \%$ ) on preference relative to the unsegmented schedule, when the early terminal-link stimulus was reinstated, and Experiment 2 tested the same effect when the early terminallink stimulus was not reinstated and was instead replaced by a new stimulus.

\section{Experiment 1}

\subsection{Materials and methods}

\subsubsection{Participants}

The participants were 32 undergraduate students ( 20 females and 12 males) at the University of Lille who were all volunteers without any compensation. They were recruited at the library of the university.

\subsubsection{Apparatus}

The study was conducted in a sound-attenuated chamber. Each participant was seated in a chair at a table on which a monitor connected to a computer was placed. All participants were trained and tested with a program created with Labview 8.0 (National Instruments Corporation, Austin, TX).

\subsubsection{Procedure}

At the start of the experiment, participants were given instructions presented on the screen generally explaining the procedure (the exact instructions are provided in Appendix A). Participants were also orally told before these instructions that the aim of the experiment was to study preference during a waiting time.

Fig. 1 illustrates schematically the general procedure. Single 15-min sessions per participant consisted of eight successive presentations of blocks of two training trials (i.e., one for each component; see Fig. 1) followed by one test trial, resulting in 24-trial sessions. As shown in Fig. 1, on training trials, the initial link, represented by a circle colored in white, was presented at the left

\section{Training Trials}

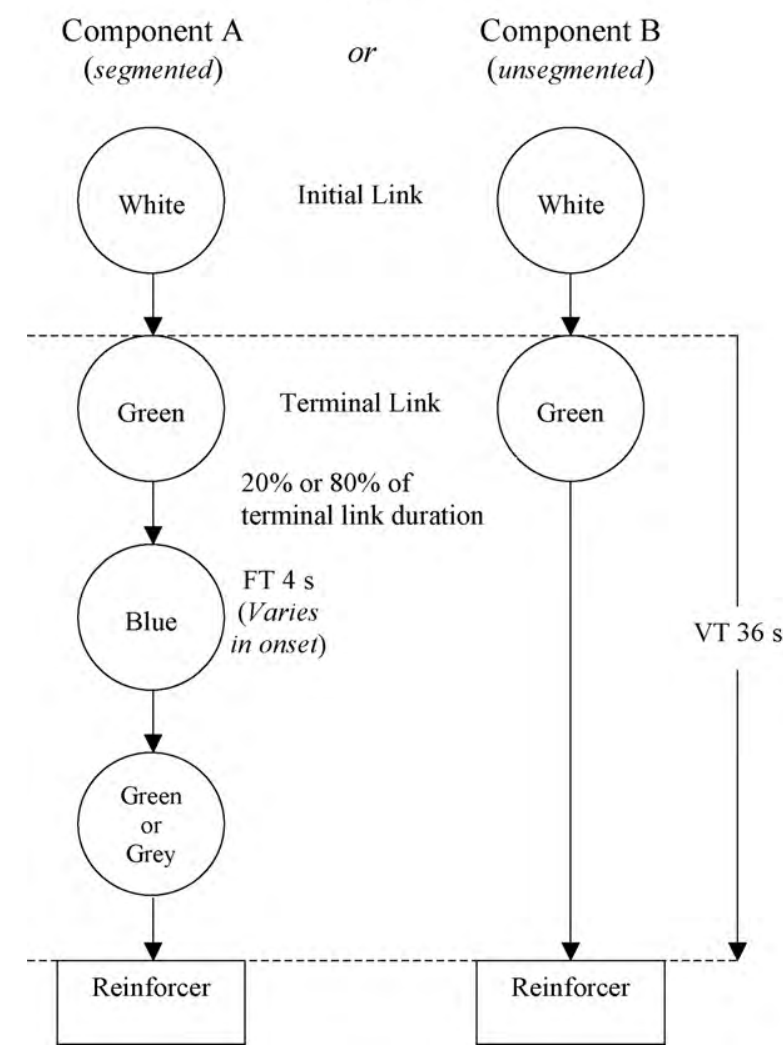

Fig. 1. Schematic representation of the experimental procedure in training trials in Experiments 1 and 2 . The left portion of the figure represents the segmented component and indicates the sequence of events when the participant clicked once on the left key during the initial links (the circle was colored white and one click to it activated the terminal-link) The right portion represents the unsegmented component and indicates the sequence of events when the participant clicked once to the right circle in initial link. The segmented schedule (Component A) consisted of a presentation of a brief S+ stimulus (i.e., blue) after $20 \%$ or $80 \%$ of the terminal-link duration had elapsed and lasted for $4 \mathrm{~s}$ after which either the early terminal-link stimulus (i.e., green; Experiment 1) or a new stimulus was presented (i.e., grey; Experiment 2) for $x \mathrm{~s}$ until reinforcement presentation. The terminal-link duration of the segmented schedule matched the terminal-link duration of the unsegmented alternative. The presentation of each component alternated. Note that after the brief S+ stimulus presentation the initial link stimulus reappeared until reinforcement onset. 
for one component and at the right of the monitor screen for the other component. One mouse click to the circle made it disappear (i.e., fixed-ratio 1, or FR1 schedule) and initiated the terminal-link stimulus that was a change in color of the circle to either blue or green (i.e., the color change was counterbalanced between subjects). The delay of reinforcement in the terminal-links was the same for both components, that is, a variable delay with a mean of $36 \mathrm{~s}$ (variable-time $36 \mathrm{~s}$, or VT $36 \mathrm{~s}$ schedule). The distribution of delays for the variable schedule was $24,32,40$, and 48 s (equiprobable, mean $=36 \mathrm{~s}$ ) and the scheduled delay was always the same within a block of two components and for the test trial. Each delay value was presented twice in a random order (four values, presented twice each, for a total of eight trials for each of the two components). For both components, reinforcement consisted of a 3-s presentation of a preferred picture chosen initially by the participant among 15 available pictures or drawings (the 15 preselected as desirable to look at by several males and females, the pictures are available from the first author). The terminal-links differed between components in whether or not the color of the circle changed during the terminal-link. Specifically, in the unsegmented schedule (Component B in Fig. 1), the same color was present until reinforcement delivery (i.e., blue or green, counterbalanced between participants). In the segmented schedule (Component $\mathrm{A}$ in Fig. 1), the color changed from blue to green, or green to blue (again, the stimulus change was counterbalanced across participants) for $4 \mathrm{~s}$ after $19.2 \mathrm{~s}, 25.6 \mathrm{~s}, 32.0 \mathrm{~s}$, or $38.4 \mathrm{~s}$ of the terminal-link duration had elapsed ( $80 \%$ of the variable terminal-link durations of $24,32,40$, and $48 \mathrm{~s}$, respectively. As described below, a $20 \%$ condition was also conducted.) Then the original terminal-link stimulus returned and was presented for the remaining time of the scheduled terminal-link. The order of training trial type (i.e., segmented or unsegmented component) was randomized for each participant, and the position of the segmented vs unsegmented outcome was counterbalanced across participants. On test trials participants were asked a single question: "Which side do you prefer? Click left or right."

The different groups will now be described. Conditions only differed with respect to Component A (see Fig. 1). For each condition, 14 different participants were recruited and tested in a betweensubject design.

$80 \%$ reinstatement. In Component $\mathrm{A}$, the 4 -s stimulus was presented after $80 \%$ of the terminal-link duration had elapsed, then the original or early terminal-link stimulus was reinstated and was presented until reinforcer delivery.

$20 \%$ reinstatement. In Component $\mathrm{A}$, the 4 -s stimulus was presented after $20 \%$ of the terminal-link duration had elapsed, then the early terminal-link stimulus was reinstated and was presented until reinforcer delivery.

\subsubsection{Follow up}

For four additional participants, the same experimental procedure as the $80 \%$ reinstatement was used except that the number of blocks was increased from 8 to 10 , and five instead of one session were conducted per participant, in order to increase the stability and reliability of the results that were previously collected in a single session. The left/right position of Components A and B (see Fig. 1) were randomized between sessions and also the color of stimulus change (blue to green, or green to blue). All other aspects remained unchanged.

\subsection{Results and discussion}

As it can be seen in Table 1, the results show that for the $80 \%$ reinstatement group, most of the participants (i.e., 12 of 14) preferred strongly the segmented component $(M=85.71 \%$; above $50 \%$ indicates preference). Whereas, for the $20 \%$ reinstatement group,
Table 1

The individual percentage of choice for the segmented schedule in the betweensubject conditions of Experiment 1 (not included the within-subject follow up results) and of Experiment 2. The group mean percentage of choice and standard error mean for each phase are also shown.

\begin{tabular}{|c|c|c|c|}
\hline \multicolumn{4}{|l|}{ Condition } \\
\hline \multicolumn{2}{|l|}{ Experiment 1} & \multicolumn{2}{|l|}{ Experiment 2} \\
\hline $\begin{array}{l}80 \% \\
\text { reinstatement }\end{array}$ & $\begin{array}{l}20 \% \\
\text { reinstatement }\end{array}$ & $\begin{array}{l}80 \% \text { no } \\
\text { reinstatement }\end{array}$ & $\begin{array}{l}20 \% \text { no } \\
\text { reinstatement }\end{array}$ \\
\hline 100 & 0 & 75 & 25 \\
\hline 100 & 25 & 50 & 75 \\
\hline 50 & 25 & 25 & 0 \\
\hline 100 & 100 & 50 & 100 \\
\hline 100 & 100 & 100 & 100 \\
\hline 50 & 25 & 100 & 25 \\
\hline 100 & 50 & 75 & 50 \\
\hline 100 & 0 & 50 & 0 \\
\hline 75 & 25 & 100 & 0 \\
\hline 75 & 100 & 75 & 25 \\
\hline 75 & 25 & 75 & 100 \\
\hline 100 & 25 & 75 & 100 \\
\hline 75 & 100 & 25 & 50 \\
\hline 100 & 0 & 75 & 100 \\
\hline 85.71 & 42.86 & 67.86 & 53.57 \\
\hline 5.05 & 10.62 & 6.65 & 11.07 \\
\hline
\end{tabular}

no systematic preference for any schedule was found $(M=45.83 \%)$. The difference between the $80 \%$ reinstatement group and the $20 \%$ reinstatement group in choices of the segmented outcome was significant, $\chi^{2}(1,14)=9.33, p<.001$. These results showed a preference for the segmented schedule only when the $\mathrm{S}+$ appeared closer to reinforcement. This outcome is predicted by delay-reduction theory because the value of a stimulus depends on the amount of delay reduction it signals (Fantino, 1969). In effect, the $S+$ in the 80\% reinstatement group was relatively closer to reinforcement compared to the one in the $20 \%$ reinstatement group that signaled a small delay reduction compared to the terminal-link stimulus in the unsegmented component.

As it can be seen in Fig. 2, the results show relatively stable preference for the segmented outcome sessions (i.e., above 50\%) over five sessions for all participants, except for P2 in which a shift in preference toward the segmented side was observed at the fourth session, and P1 in session 2. Furthermore the other participants showed a clear preference for the segmented alternative from the first session. Finally in most of participants neither the side of the segmented alternative nor the colors associated with the segmented schedule affected the choice pattern. These results are important for two reasons: the first is that it replicated the observed preference for the segmented schedule in the $80 \%$ rein-

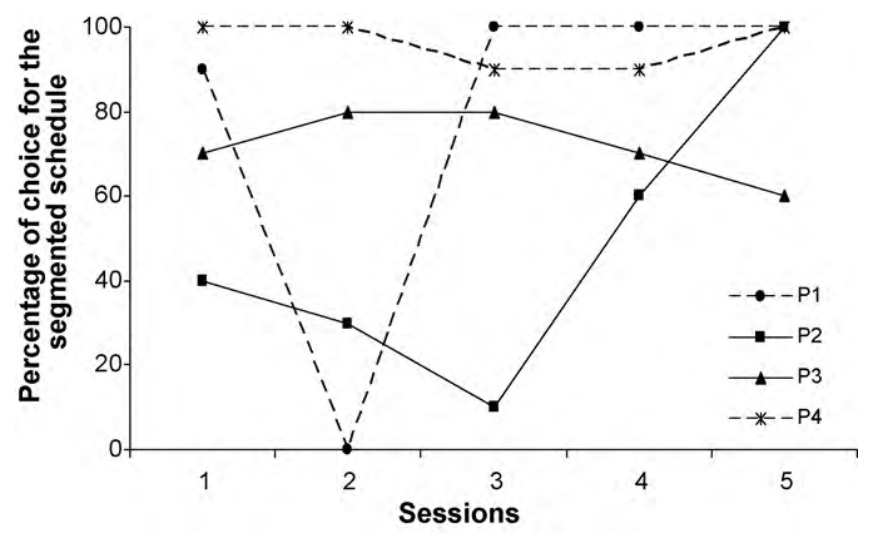

Fig. 2. Individual choice proportion for the segmented VT schedule in the follow up condition for five sessions. 
statement group. The second reason is that preference scores were stable and reliable despite changes in procedure arrangements between sessions as the side and the colors associated with the segmented vs unsegmented schedule (it means that in this procedure, preference was not biased by the position and the specific colors). So preference scores collected in the first session should be a good indicator of preference scores after up to fourth sessions or under testing on different stimulus conditions. Consequently here, it seems to be well-adapted to test human participants under a single session and without counterbalancing side and color associated with the segmented vs unsegmented schedule, since participants tested here behaved similarly to those in the between-subject design.

\section{Experiment 2}

In Experiment 2, the purpose was to investigate the idea that preference for the segmented schedule observed in Experiment 1 was also affected by the reinstatement of the early stimulus, in addition to the increase in delay reduction signaled by the $S+$ stimulus. To address this issue, two parallel conditions of Experiment 1 (segmentation at $20 \%$ and at $80 \%$ ) were implemented in which the early terminal-link stimulus on the segmented component was not reinstated following the $\mathrm{S}+$ presentation. If absence of preference for the segmented schedule, observed in the literature, was due to the absence of contiguity with reinforcement for the early terminal-link stimulus, then we should observe the same outcome here when a novel stimulus was presented following the $\mathrm{S}+$, one that also was contiguous with reinforcement. So, no difference in preference for the segmented outcome should be expected within the same group ( $20 \%$ and $80 \%$ delay reduction).

\subsection{Materials and methods}

\subsubsection{Participants and apparatus}

The participants were 28 undergraduate students (18 females and 10 males) at the University of Lille who were all volunteers without any compensation. They were recruited at the library of the university. The apparatus was the same as the one used in Experiment 1.

\subsubsection{Procedure}

The procedure was similar as the one described in Experiment 1 except that in Component A (see Fig. 1), a new stimulus (i.e., grey circle) was inserted, following the $\mathrm{S}+$ presentation, that replaced the reinstatement of the early terminal-link stimulus in Experiment 1. All other aspects remained unchanged.

\subsection{Results and discussion}

Results showed that most participants (i.e., 9 of 14) preferred the segmented alternative in the $80 \%$ no reinstatement group ( $M=67.86 \%$; above $50 \%$ indicates preference), and that in the $20 \%$ no reinstatement group, no systematic preference for any alternative was observed (i.e., 6 of 14 preferred the segmented component, and 6 of 14 preferred the unsegmented component). However, unlike Experiment 1, there is no reliable difference between the $80 \%$ no reinstatement group and the $20 \%$ no reinstatement group in choices of the segmented outcome, $\chi^{2}(1,14)=1.29, p=.2556$.

\section{General discussion}

The present results show that humans reliably preferred a VT segmented schedule over an equivalent VT unsegmented schedule when the S+ stimulus of the segmented schedule was presented briefly and was correlated with a $80 \%$ of delay reduction to reinforcement. These results are in conflict with those collected with a comparable experimental procedure in which preference for the unsegmented schedule was typically found (e.g., Leung, 1993). In the introduction, we asked which variable controls preference for an unsegmented schedule, as is commonly observed in the literature observed in literature. Three hypotheses were proposed. The first one, NSH, required preference to be an inverse function of the number of segments in the outcome. The second hypothesis, $\mathrm{RH}$, was to explain the greater conditioned value of the unsegmented component by the fact that its terminal-link stimulus was contiguous with reinforcement unlike the early terminal-link stimulus of the segmented outcome. Therefore, the early terminal-link stimulus was viewed in the literature as an S-Delta (e.g., Duncan and Fantino, 1972). According to the third hypothesis, DRT, when the component is segmented in half, the S+ stimulus is not associated with sufficient delay reduction to be preferred over the unsegmented outcome (even if it signals a greater delay reduction relative to the terminal-link stimulus of the unsegmented component). So if the S+ stimulus was presented closer to reinforcement, then it could increase preference for the segmented alternative. In fact the present results give some support to both the reinstatement hypothesis and to DRT. Note, that the two hypotheses are not mutually exclusive and may interact in their effect on preference. In effect, for the reinstatement hypothesis, within the conditions in which in the segmented schedule the S+ stimulus was presented after $80 \%$ of the terminal-link had elapsed, we observed preference for the segmented schedule when the early terminal-link stimulus was also contiguous with reinforcement delivery (i.e., was reinstated after the $S+$ presentation) (Experiment 1) and no significant preference when it was not (Experiment 2, even if a small preference for the segmented component was observed). But the difference in preference could not be accounted for solely by the reinstatement variable as we found no preference for the segmented schedule when the S+ stimulus occurred after $20 \%$ of the terminal-link duration although the early terminal-link stimulus also returned and was contiguous with reinforcement delivery. For the delay-reduction hypothesis, the variable that could explain the shift of preference was likely the manipulated delay between $\mathrm{S}+$ onset and reinforcement delivery in the segmented schedule (i.e., the degree of reduction in delay of reinforcement the stimulus signals). In effect, we observed preference for the segmented schedule only when the S+ stimulus was correlated with a relatively great delay reduction to reinforcement as predicted by DRT (Fantino, 1969, 2008). A similar shift of preference toward the segmented alternative or at least a decrease of preference for the unsegmented outcome was also found in other studies when the interval between the second terminal-link stimulus onset and reinforcement delivery was decreased (Leung and Winton, 1986, 1988). In terms of the three hypotheses discussed in the introduction (reinstatement, number of segments, and DRT), and the ranked preference they predict, only DRT provided an approximation to the obtained preferences. It appears that DRT, modulated by the influence of reinstatement, captures the preference ordering obtained.

To conclude, first, the results collected here indicate that in certain conditions we observed preference for a schedule that included a brief stimulus correlated with a substantial (80\%) reduction in time to reinforcement. Whereas superimposed brief stimuli have often failed to affect preference, the stimuli from prior studies were not correlated with delay reduction (e.g., Schuster, 1969; see Fantino, 2008, for a review). Second, when the stimulus present at the onset of a segmented outcome is the same as the one present at the onset of reinforcement, the well-established preference for unsegmented over segmented outcomes was not observed. 


\section{Acknowledgements}

We thank the reviewers for helpful comments, providing language assistance, and writing assistance.

\section{Appendix A. Appendix A}

The oral instructions given at the beginning of the experiment was the following:

"You will see the picture you have selected on the monitor screen. To see the picture, you have to click on one circle that will be presented at the left or right of the screen. You will then have to wait a few seconds before you see the picture. During this time please remain focused on the circle because different events will appear on the different circles. Please, don't count the time during the waiting period." (this was because subjects could discover by counting that both outcomes last actually the same time).

\section{References}

Autor, S.M., 1960. The strength of conditioned reinforcers as a function of frequency and probability of reinforcement. Unpublished Doctoral Dissertation. Harvard University.
Autor, S.M., 1969. The strength of conditioned reinforcers as a function of frequency and probability of reinforcement. In: Hendry, D.P. (Ed.), Conditioned Reinforcement. The Dorsey Press, Homewood, IL, pp. 127-162.

Duncan, B., Fantino, E., 1972. The psychological distance to reward. J. Exp. Anal. Behav. 18, 23-34.

Fantino, E., 1969. Choice and rate of reinforcement. J. Exp. Anal. Behav. 12, 723 730.

Fantino, E., 2008. Choice, conditioned reinforcement, and the Prius effect. Behav. Analyst 31, 95-111.

Herrnstein, R.J., 1964. Secondary reinforcement and rate of primary reinforcement J. Exp. Anal. Behav. 7, 27-36.

Leung, J.P., Winton, A.S.W., 1986. Preference for less segmented schedules fixedtime components in concurrent-chain schedules of reinforcement. J. Exp. Anal. Behav. 46, 175-183.

Leung, J.P., Winton, A.S.W., 1988. Preference for simple interval schedules of reinforcement in concurrent chains: effect of segmentation ratio. J. Exp. Anal. Behav. 49, 9-20.

Leung, J.P., 1989. Psychological distance to reward: a human replication. J. Exp. Anal. Behav. 51, 343-352.

Leung, J.P., 1993. Psychological distance to reward: segmentation of aperiodic schedules of reinforcement. J. Exp. Anal. Behav. 59, 401-410.

Schuster, R.H., 1969. The strength of conditioned reinforcers as a function of frequency and probability of reinforcement. In: Hendry, D.P. (Ed.), Principles of Behavior. Dorsey Press. 